\title{
Pseudo Control Hedging and its Application for Safe Flight Envelope Protection
}

\author{
T.J.J. Lombaerts*, \\ Delft University of Technology, P.O. Box 5058, 2600 GB Delft, The Netherlands \\ G.H.N. Looye ${ }^{\dagger}$ \\ German Aerospace Center DLR, Münchner Straße 20, 82234 Wessling, Germany \\ Q.P. Chu \\ Delft University of Technology, P.O. Box 5058, 2600 GB Delft, The Netherlands
}

\begin{abstract}
This paper describes how the previously developed concept of Pseudo Control Hedging (PCH) can be integrated in a Fault Tolerant Flight Controller (FTFC) as a safe flight envelope protection system of the first degree. This PCH algorithm adapts the reference model for the system output in case of unachievable commands due to control input saturation. As an example, this algorithm has been applied in the pitch rate and velocity control loops of a high fidelity Boeing 747 simulation model where its beneficial influence has been illustrated. The nonlinear adaptive control law used for this example is a triple layered nonlinear dynamic inversion algorithm, based upon the concept of time scale separation.
\end{abstract}

\section{Nomenclature}

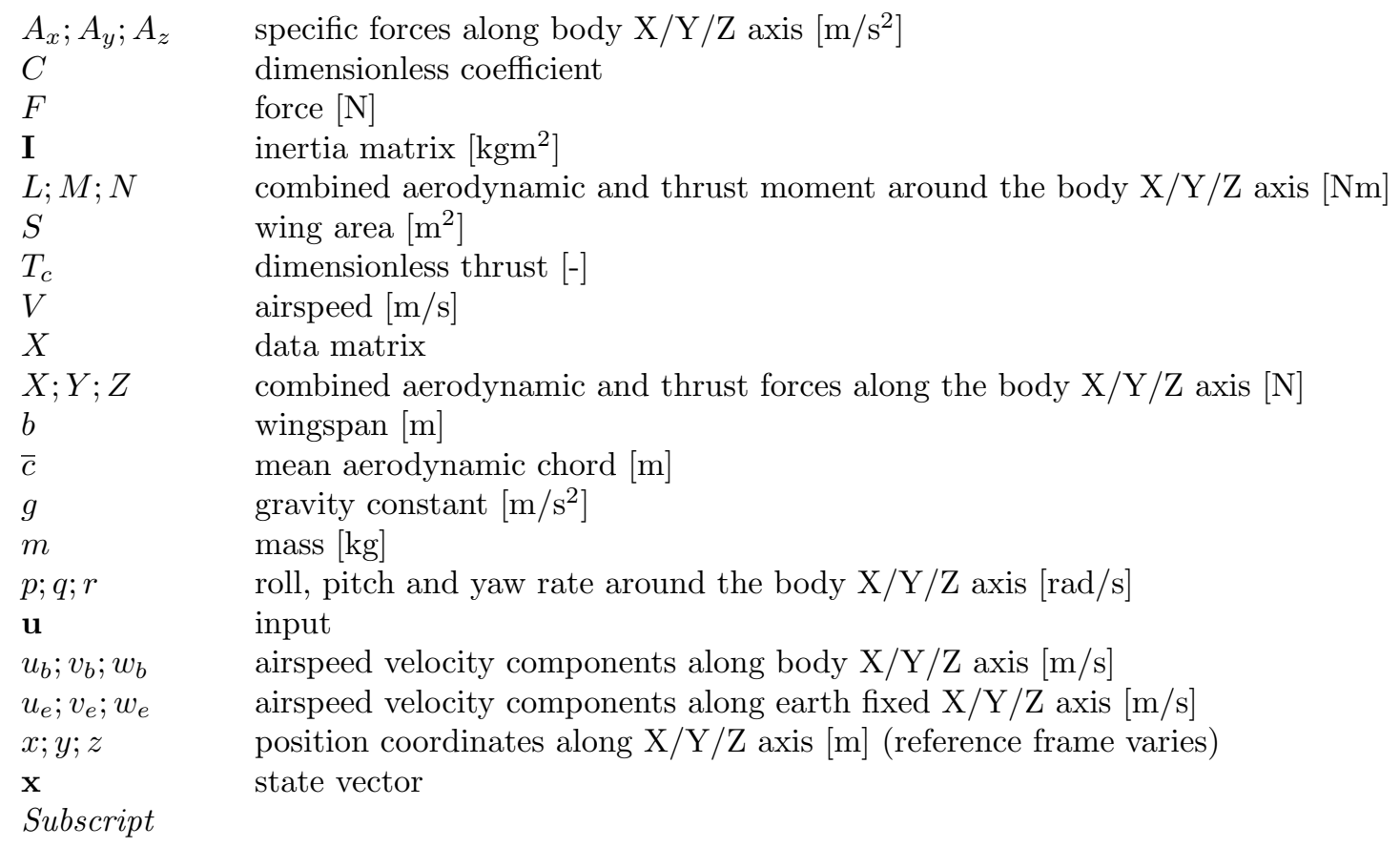

\footnotetext{
${ }^{*}$ Researcher and lecturer, Control and Simulation Division, Faculty of Aerospace Engineering, e-mail: t.j.j.lombaerts@tudelft.nl, AIAA student member

$\dagger$ Researcher, Institute of Robotics and Mechatronics, Department of System Dynamics and Control, AIAA member

$¥$ Associate professor, Control and Simulation Division, Faculty of Aerospace Engineering, AIAA member

$\S$ Professor, Control and Simulation Division, Faculty of Aerospace Engineering, AIAA member
} 


$\begin{array}{ll}0 & \text { constant term } \\ A & \text { with respect to aerodynamic reference frame } \\ b & \text { with respect to body fixed reference frame } \\ e & \text { with respect to earth fixed reference frame } \\ k & \text { with respect to kinematic reference frame } \\ a ; e ; r & \text { aileron, elevator and rudder } \\ \text { comm } & \text { commanded } \\ f_{o} ; f_{i} & \text { outer, inner flaps } \\ l ; m ; n & \text { combined aerodynamic and thrust moment around the body } \mathrm{X} / \mathrm{Y} / \mathrm{Z} \text { axis }[\mathrm{Nm}] \\ l ; r & \text { left,right } \\ T A S & \text { true airspeed } \\ r u ; r l & \text { upper and lower rudders } \\ \text { sp } & \text { spoiler } \\ \text { air } ; \text { ail } ; \text { aor } ; \text { aol } & \text { inner right, inner left, outer right and outer left ailerons } \\ \text { eir } ; \text { eil; } \text { eor } ; \text { eol } & \text { inner right, inner left, outer right and outer left elevators } \\ \text { Symbol } & \\ \alpha ; \beta ; \gamma & \text { angle of attack, sideslip angle and flightpath angle [rad] } \\ \delta & \text { control surface deflection [rad] } \\ \mu & \text { aerodynamic roll angle [rad] } \\ \nu & \text { virtual input } \\ \rho & \left.\text { air density [kg/m }{ }^{3}\right] \\ \phi ; \theta ; \psi & \text { roll, pitch and yaw angle [rad] } \\ \chi & \text { course angle [rad] }\end{array}$

\section{Introduction}

$\mathrm{B}_{\text {EING inspired by some recent aircraft accidents, fault tolerant flight control (FTFC) is a control discipline }}$

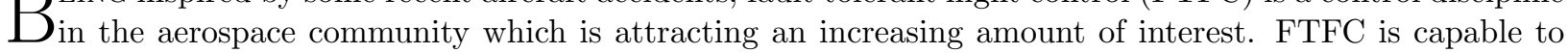
reduce the number of aircraft accidents caused by loss of control (LOC) in flight. ${ }^{1-7}$ One of the methods to achieve fault tolerant control is by applying so-called indirect adaptive control, where a model based adaptive control algorithm relies upon a real-time updated aerodynamic model of the damaged aircraft. Examples of this control method are nonlinear dynamic inversion, ${ }^{8}$ backstepping ${ }^{9}$ or model predictive control. ${ }^{10,11}$ This publication will focus on the nonlinear dynamic inversion based approach.

A major attraction of dynamic inversion is its ability to naturally handle changes of operating condition, which removes the need for gain scheduling, e.g. for classical control methods. This is especially advantageous for control of space re-entry vehicles, due to their extreme and wide operating conditions which vary between supersonic speed during re-entry and subsonic regions during the terminal glide approach phase to the runway. Another advantage is its natural property of decoupling the control axes, i.e. no coupling effects remain between steering channels and the different degrees of freedom. NDI control has been implemented in the Lockheed F-35 Lightning II. ${ }^{12,13}$ Dynamic inversion is a popular control method for flight control and aircraft guidance ${ }^{14-19}$ as well as reconfiguring control. ${ }^{20-23}$

Another important issue is Pseudo Control Hedging. ${ }^{24,25}$ This adapts the reference model for the system output in case of unachievable commands due to control saturation. This is worthwhile to include in addition to the dynamic inversion based controller, since it provides some partial form of flight envelope protection, and has some similarity with an anti-windup scheme.

The combined setup of NDI and PCH has been evaluated on a high fidelity Pratt and Whitney powered Boeing 747-100/200 simulation model. A detailed nonlinear simulation model of this aircraft is available from the National Aerospace Laboratory NLR. This RECOVER (REconfigurable COntrol for Vehicle Emergency Relief) benchmark model is discussed in detail by Smaili et $\mathrm{al}^{26,27}$ and has been used (also in earlier versions) by a number of investigators and organizations. ${ }^{10,28,29}$ Other fault tolerant flight control strategies have been applied to the same benchmark model. ${ }^{30-34}$ A more extensive source of information can be found in the literature. ${ }^{35}$ This RECOVER model has also been used for previous fault tolerant flight control research 
activities in the same research project. ${ }^{8,36,37}$

This paper will discuss first the implementation of nonlinear dynamic inversion in section II. This setup is based upon three consecutive NDI loops, based upon the principle of time scale separation. Subsequently, section III provides a brief technical discussion of the Pseudo Control Hedging Principle. Two applications of NDI and PCH combined are elaborated in section IV. Finally, conclusions and remarks can be found in section V.

\section{Nonlinear Dynamic Inversion control setup}

First, the concept of dynamic inversion will be introduced by means of the concept of Lie derivatives. Then, the concept of multiloop NDI is elaborated by means of the time scale separation principle. Thereafter, the three NDI loops are elaborated successively.

\section{A. The concept of NDI}

The general idea of nonlinear dynamic inversion is as follows. Consider the nonlinear MIMO system dynamic model, which is assumed to be affine in the input:

$$
\dot{\mathbf{x}}=\mathbf{f}(\mathbf{x})+\mathbf{G}(\mathbf{x}) \mathbf{u}
$$

The output $\mathbf{y}$ of the system is then expressed as a function $\mathbf{h}$ of the aircraft state vector $\mathbf{x}$ :

$$
\mathbf{y}(\mathbf{x})=\mathbf{h}(\mathbf{x})
$$

Defining the matrix $\nabla \mathbf{h}(\mathbf{x})$ as the Jacobian matrix:

$$
\frac{\partial \mathbf{h}(\mathbf{x})}{\partial \mathbf{x}}=\nabla \mathbf{h}(\mathbf{x})
$$

the time derivatives of the outputs in eq. (2) can be expressed as:

$$
\frac{d \mathbf{y}}{d t}=\nabla \mathbf{h}(\mathbf{x})[\mathbf{f}(\mathbf{x})+\mathbf{G}(\mathbf{x}) \mathbf{u}]=L_{f}^{1} \mathbf{h}(\mathbf{x})+L_{g} \mathbf{h}(\mathbf{x}) \mathbf{u}
$$

where $L_{f}^{1} \mathbf{h}(\mathbf{x})=\nabla \mathbf{h}(\mathbf{x}) \mathbf{f}(\mathbf{x})$ denotes the first order Lie derivative vector and the $L_{g} \mathbf{h}(\mathbf{x})=\nabla \mathbf{h}(\mathbf{x}) \mathbf{G}(\mathbf{x})$. If the second term of eq. (4) is zero, more time derivatives of eq. (4) are required, generally until the second term of eq. (4) is nonzero. This nonzero time derivative order is defined as "relative degree". In general, as the elements within the output vector $\mathbf{y}(\mathbf{x})$ may have different relative degrees, it is convenient to write the time derivative for each output as:

$$
\frac{d^{r_{i}} y_{i}}{d t^{r_{i}}}=\frac{d^{r_{i}} h_{i}(\mathbf{x})}{d t^{r_{i}}}=L_{f}^{r_{i}} h_{i}(\mathbf{x})+\sum_{j=1}^{m} L_{g_{j}} L_{f}^{r_{i}-1} h_{i}(\mathbf{x}) u_{j}
$$

In eq. (5), $r_{i}$ is the relative degree for the $i^{\text {th }}$ output. A collection of all differentiated $\left(r_{i}^{\text {th }}\right.$ order) outputs yields:

$$
\mathbf{y}^{r}(\mathbf{x})=\mathbf{l}(\mathbf{x})+\mathbf{M}(\mathbf{x}) \mathbf{u}
$$

with:

$$
\begin{gathered}
\mathbf{y}^{r}(\mathbf{x})=\left[\begin{array}{c}
\frac{d^{r_{1}} h_{1}(\mathbf{x})}{d t^{r}} \\
\vdots \\
\frac{d^{r} h_{m}(\mathbf{x})}{d t^{r_{m}}}
\end{array}\right] \\
\mathbf{l}(\mathbf{x})=\left[\begin{array}{c}
L_{f}^{r_{1}} h_{1}(\mathbf{x}) \\
L_{f}^{r_{2}} h_{2}(\mathbf{x}) \\
\vdots \\
L_{f}^{r_{m}} h_{m}(\mathbf{x})
\end{array}\right]
\end{gathered}
$$

3 of 17 
and

$$
\mathbf{M}(\mathbf{x})=\left[\begin{array}{cccc}
L_{g_{1}} L_{f}^{r_{1}-1} h_{1}(\mathbf{x}) & L_{g_{2}} L_{f}^{r_{1}-1} h_{1}(\mathbf{x}) & \cdots & L_{g_{m}} L_{f}^{r_{1}-1} h_{1}(\mathbf{x}) \\
L_{g_{1}} L_{f}^{r_{2}-1} h_{2}(\mathbf{x}) & L_{g_{2}} L_{f}^{r_{2}-1} h_{2}(\mathbf{x}) & \cdots & L_{g_{m}} L_{f}^{r_{f}-1} h_{2}(\mathbf{x}) \\
\vdots & \vdots & \vdots & \vdots \\
L_{g_{1}} L_{f}^{r_{m}-1} h_{m}(\mathbf{x}) & L_{g_{2}} L_{f}^{r_{m}-1} h_{m}(\mathbf{x}) & \cdots & L_{g_{m}} L_{f}^{r_{m}-1} h_{m}(\mathbf{x})
\end{array}\right]
$$

Solving for $\mathbf{u}$ if the total relative degree $r=r_{1}+r_{2}+\ldots+r_{m}=n$, with $n$ the number of states of the system, by introducing a virtual outer loop control input vector $\boldsymbol{\nu}$, which consists of time derivatives of control variables $c v_{i}(\mathbf{x})$ up to the corresponding relative degree $r_{i}$ :

$$
\mathbf{u}=\mathbf{M}^{-1}(\mathbf{x})[\boldsymbol{\nu}-\mathbf{l}(\mathbf{x})]
$$

with:

$$
\boldsymbol{\nu}(\mathbf{x})=\left[\begin{array}{c}
\frac{d^{r_{1}} c v_{1}(\mathbf{x})}{d t^{r_{1}}} \\
\vdots \\
\frac{d^{r} m}{d t^{r_{m}}(\mathbf{x})}
\end{array}\right]
$$

then this results in a closed-loop system with a linear and decoupled input-output relation:

$$
\mathbf{y}^{r}(\mathbf{x})=\left[\begin{array}{c}
\frac{d^{r_{1}} h_{1}(\mathbf{x})}{d t^{r_{1}}} \\
\vdots \\
\frac{d^{r m} h_{m}(\mathbf{x})}{d t^{r_{m}}}
\end{array}\right]=\boldsymbol{\nu}=\left[\begin{array}{c}
\frac{d^{r_{1}} c v_{1}(\mathbf{x})}{d t^{r_{1}}} \\
\vdots \\
\frac{d^{r m_{c}} v_{m}(\mathbf{x})}{d t^{r}{ }^{\prime}}
\end{array}\right]
$$

Thus the control law for tracking tasks

$$
\frac{d^{r_{i}} c v_{i}}{d t^{r_{i}}}=\frac{d^{r_{i}} h_{i_{d}}}{d t^{r_{i}}}-k_{0_{i}} e-k_{1_{i}} \dot{e}-\ldots-k_{\left(r_{i}-1\right)_{i}} e^{\left(r_{i}-1\right)} \text { with } e=y_{i_{d}}(t)-y_{i}(t)
$$

for $i=1, \ldots, m$ with the $k_{j}$ s chosen so that $p^{n}+k_{n-1} p^{n-1}+\ldots+k_{1} p$ is a stable polynomial, leads to the exponentially stable tracking dynamics for $i=1, \ldots, m$ :

$$
e^{\left(r_{i}\right)}+k_{\left(r_{i}-1\right)_{i}} e^{\left(r_{i}-1\right)}+\ldots+k_{1_{i}} \dot{e}+k_{0_{i}} e=0 \text { with } e(t) \rightarrow 0
$$

By making use of Nonlinear Dynamic Inversion (NDI), the nonlinear aircraft dynamics can be cancelled out such that the resulting system behaves like a pure single $r$-th order integrator. In eq. (10), $\mathbf{l}(\mathbf{x})$ represents the airframe/engine model and $\mathbf{M}(\mathbf{x})$ is the so-called effector blending model. Note that the effector blending model $\mathbf{M}(\mathbf{x})$ needs to be inverted. More information is available in the literature. ${ }^{38,39}$

\section{B. Multiloop NDI and time scale separation}

In this setup, the multiloop NDI concept has been applied, based on time scale separation. The time scale separation principle states the following. When a moment acts on an object, then primarily the angular rates change, whereas the attitude angles remain approximately the same for small time steps. This concept is commonly used in aircraft control, for example in Reiner et al. ${ }^{15}$ As a consequence of the time scale separation principle, it is sufficient for each subsystem to consider the first order Lie derivative to find the relevant control input, and the local "relative degree" is one. In theory, this separation principle involves some stability issues, however, practice has shown that the bandwidths of angular rates and attitude angles are sufficiently separated to prevent the risk for instabilities due to interactions. In the remainder of the discussions, first order systems will be considered.

As a matter of fact, the nonlinear dynamic inversion control setup can be defined in various alternative ways. One way is the implementation of a double NDI layer. ${ }^{8}$ An alternative is the use of a triple layered NDI setup. ${ }^{40}$ 


\section{First dynamic inversion loop: angular rates}

A similar structure as described in eq. (10) can be found in the inner loop for angular rate control: ${ }^{8}$

$$
\begin{aligned}
{\left[\begin{array}{c}
\delta_{a} \\
\delta_{e} \\
\delta_{r}
\end{array}\right]=} & {\left[\begin{array}{ccc}
b \tilde{C}_{l_{\delta_{a}}} & 0 & b \tilde{C}_{l_{\delta_{r}}} \\
0 & \bar{c} \tilde{C}_{m_{\delta_{e}}} & 0 \\
b \tilde{C}_{n_{\delta_{a}}} & 0 & b \tilde{C}_{n_{\delta_{r}}}
\end{array}\right]^{-1} } \\
& \left\{\frac{\mathbf{I}}{\frac{1}{2} \rho V^{2} S}\left(\left[\begin{array}{c}
\nu_{p} \\
\nu_{q} \\
\nu_{r}
\end{array}\right]+\mathbf{I}^{-1}\left[\begin{array}{c}
p \\
q \\
r
\end{array}\right] \times\left(\mathbf{I}\left[\begin{array}{l}
p \\
q \\
r
\end{array}\right]\right)-\left[\begin{array}{c}
b C_{l_{\text {states }}} \\
\bar{c} C_{m_{\text {states }}} \\
b C_{n_{\text {states }}}
\end{array}\right]\right\}\right.
\end{aligned}
$$

where the virtual inputs $\left[\begin{array}{lll}\nu_{p} & \nu_{q} & \nu_{r}\end{array}\right]^{T}$ are the time derivatives of the rotational rates of the aircraft, which are selected to be the control variables in order to obtain rate control. The first part of eq. (15) performs the control inversion, while the second part contains the state inversion. Moreover, control effectivity is defined as follows:

$$
\begin{aligned}
\tilde{C}_{l_{\delta_{a}}} & =-C_{l_{\delta_{a_{i r}}}}+C_{l_{\delta_{a_{i l}}}}-C_{l_{\delta_{a_{o r}}}}+C_{l_{\delta_{a_{o l}}}}-C_{l_{\delta_{s p_{1}}}}-\ldots-C_{l_{\delta_{s p_{5}}}}+C_{l_{\delta_{s p} p_{8}}}+\ldots+C_{l_{\delta_{s p_{1}}}} \\
\tilde{C}_{n_{\delta_{a}}} & =-C_{n_{\delta_{a_{i r}}}}+C_{n_{\delta_{a_{i l}}}}-C_{n_{\delta_{a_{o r}}}}+C_{n_{\delta_{a_{o l}}}}-C_{n_{\delta_{s p_{1}}}}-\ldots-C_{n_{\delta_{s p_{5}}}}+C_{n_{\delta_{s p_{8}}}}+\ldots+C_{n_{\delta_{s p_{12}}}} \\
\tilde{C}_{m_{\delta_{e}}} & =C_{m_{\delta_{e_{i r}}}}+C_{m_{\delta_{e_{i l}}}}+C_{m_{\delta_{e_{o r}}}}+C_{m_{\delta_{e_{o l}}}} \\
\tilde{C}_{l_{\delta_{r}}} & =C_{l_{\delta_{r_{u}}}}+C_{l_{\delta_{r_{l}}}} \\
\tilde{C}_{n_{\delta_{r}}} & =C_{n_{\delta_{r_{u}}}}+C_{n_{\delta_{r_{l}}}}
\end{aligned}
$$

The different aileron, elevator, rudder and spoiler surfaces are coupled and deflect in a fixed coordinated way, as illustrated by eq. (16) through (20). All four ailerons and ten spoilers deflect simultaneously. The same holds for the four elevator surfaces and the upper and lower rudder. The development of a more flexible control allocation algorithm is part of current work. Nevertheless, the results shown here prove that this simplification has no serious detrimental effect on the performance of the FTFC module.

The weakness of classical NDI, its sensitivity to modeling errors which leads to erroneous inversion, and thus a possibly unstable result, is circumvented here by making use of the real time identified physical model, which has a greater accuracy than an off-line model. As a result, one does not only obtain an adaptive NDI routine which renders the aircraft behavior like a pure integrator in nominal situations. In failure situations, the modified aircraft model is identified by the two step method and immediately applied in the model-based adaptive NDI routine, which allows reconfiguring for the failure in real time. For more information about real time identification, see the literature. ${ }^{8,37}$

The inner loop thus focuses on pure body fixed angular rate control as elaborated in equation (15).

\section{Second dynamic inversion loop: aerodynamic angles}

The second level of the NDI loop can be set up in a similar way as the first level, this time for the aerodynamic angles $\phi, \alpha$ and $\beta$.

First, in order to obtain roll angle control, an equation needs to be found which expresses the change in roll angle in terms of the required rotational rates. Mulder et $\mathrm{al}^{41}$ provides:

$$
\frac{d \phi}{d t}=\dot{\phi}=p+(q \sin \phi+r \cos \phi) \tan \theta
$$

Separating the rotational rates $\left[\begin{array}{lll}p & q & r\end{array}\right]^{T}$ yields:

$$
\dot{\phi}=\left[\begin{array}{lll}
1 & \sin \phi \tan \theta & \cos \phi \tan \theta
\end{array}\right]\left[\begin{array}{l}
p \\
q \\
r
\end{array}\right]
$$


With respect to the angle of attack $\alpha$, the following expression holds:

$$
\begin{aligned}
\alpha & =\arcsin \left(\frac{w_{b}}{V}\right) \\
\dot{\alpha} & =\frac{1}{\sqrt{V^{2}-w_{b}^{2}}} \dot{w}_{b}=\frac{1}{\sqrt{V^{2}-w_{b}^{2}}}\left(A_{z}+g \cos \theta \cos \phi+q u_{b}-p v_{b}\right)
\end{aligned}
$$

The control law for the sideslip angle $\beta$ can be deduced in the same way, where a relation must be found between the sideslip angle $\beta$ and the body fixed angular rates:

$$
\dot{\beta}=\frac{1}{\sqrt{V^{2}-v_{b}^{2}}}\left[A_{y}+g \cos \theta \sin \phi+w_{b} p-u_{b} r\right]
$$

The overall second level NDI control law becomes then:

$$
\begin{aligned}
{\left[\begin{array}{l}
p \\
q \\
r
\end{array}\right]=} & {\left[\begin{array}{ccc}
1 & \sin \phi \tan \theta & \cos \phi \tan \theta \\
-\frac{v_{b}}{\sqrt{V^{2}-w_{b}^{2}}} & \frac{u_{b}}{\sqrt{V^{2}-w_{b}^{2}}} & 0 \\
\frac{w_{b}}{\sqrt{V^{2}-v_{b}^{2}}} & 0 & \frac{-u_{b}}{\sqrt{V^{2}-v_{b}^{2}}}
\end{array}\right]^{-1} . } \\
& \left\{\left[\begin{array}{c}
\nu_{\dot{\phi}} \\
\nu_{\dot{\alpha}} \\
\nu_{\dot{\beta}}
\end{array}\right]-\left[\begin{array}{cc}
-\frac{1}{\sqrt{V^{2}-w_{b}^{2}}}\left(A_{z}+g \cos \theta \cos \phi\right) \\
\frac{1}{\sqrt{V^{2}-v_{b}^{2}}}\left(A_{y}+g \cos \theta \sin \phi\right)
\end{array}\right]\right\}
\end{aligned}
$$

\section{E. Third dynamic inversion loop: navigational dynamics}

The procedure used in this step is inspired by the method used by Holzapfel, ${ }^{40,}{ }^{42}$ although the application for this study implies some important deviations compared to the conventional method, since an adaptive model needs to be taken into account. Main crux in this deviating approach is that this inversion loop constitutes of two separate steps. First, the kinematics based virtual inputs are transformed towards the roll angle and the symmetric aerodynamic forces through a physically interpretable nonlinear mapping. Consequently, the aforementioned force components are translated into commanded angle of attack and dimensionless thrust values via a classical NDI-setup as used before, which involves a local gradient determination step. The derivation of these control laws is elaborated below.

\section{Nonlinear mapping of virtual inputs towards roll angle and symmetric force components}

The trajectory dynamics of the aircraft can be expressed in the so-called kinematical frame of reference, where the origin is located in the airplane center of gravity, the $X_{k}$-axis coincides with the groundspeed velocity vector, $Z_{k}$-axis lies in the plane of symmetry of the aircraft, and finally the $Y_{k}$-axis is oriented perpendicular on the plane spanned by the origin and both the $X_{k}$ and $Z_{k}$ axes.

According to this definition, the kinematic (groundspeed) velocity vector is defined as follows in this frame of reference:

$$
\mathbf{V}_{k}=\left[\begin{array}{c}
V_{k} \\
0 \\
0
\end{array}\right]
$$

This velocity vector is differentiated as follows and put equal to the sum of aerodynamic and gravity forces:

$$
\begin{aligned}
\frac{d \mathbf{V}_{k}}{d t} & =\frac{\partial \mathbf{V}_{k}}{\partial t}+\mathbf{\Omega}_{k e}^{k} \times \mathbf{V}=\left(\begin{array}{c}
\dot{V}_{k} \\
0 \\
0
\end{array}\right)+\boldsymbol{\Omega}_{k e}^{k} \times\left(\begin{array}{c}
V_{k} \\
0 \\
0
\end{array}\right) \\
& =\frac{1}{m}\left(\begin{array}{ccc}
1 & 0 & 0 \\
0 & \cos \mu & -\sin \mu \\
0 & \sin \mu & \cos \mu
\end{array}\right) \Sigma \mathbf{F}_{A_{\text {aero }}}+\boldsymbol{\Theta}_{k g}\left(\begin{array}{l}
0 \\
0 \\
g
\end{array}\right)
\end{aligned}
$$

6 of 17 


$$
\begin{aligned}
\boldsymbol{\Omega}_{k e}^{k} & =\left(\begin{array}{c}
-\sin \gamma \cdot \dot{\chi} \\
\dot{\gamma} \\
\cos \gamma \cdot \dot{\chi}
\end{array}\right) \\
\boldsymbol{\Theta}_{k g} & =\left(\begin{array}{ccc}
\cos \gamma & 0 & -\sin \gamma \\
0 & 1 & 0 \\
\sin \gamma & 0 & \cos \gamma
\end{array}\right)\left(\begin{array}{ccc}
\cos \chi & \sin \chi & 0 \\
-\sin \chi & \cos \chi & 0 \\
0 & 0 & 1
\end{array}\right)
\end{aligned}
$$

In equation (29), the rotation around the aerodynamic roll angle $\mu$ corresponds to the transformation from the aerodynamic reference frame towards the kinematic frame of reference, as illustrated in the appendix. In the same way, transformation matrix $\boldsymbol{\Theta}_{k g}$ defined in eq. (31) represents the conversion from the earth fixed frame of reference towards the same aforementioned kinematic reference frame. Finally, the angular velocity vector $\boldsymbol{\Omega}_{k e}^{k}$ in eq. (30) depicts the rotational rate of the kinematic reference frame with respect to the earth fixed reference frame, expressed in terms of the kinematic frame of reference. This expression is determined by means of two consecutive rotations, as elaborated in Mulder et al: ${ }^{41}$

1. Rotation $\chi_{k}$ kinematic azimuth angle about the $Z_{e}$ axis;

2. Rotation $\gamma_{k}$ kinematic flight path angle about the $Y_{k}$ axis.

Expressed in matrix form as follows:

$$
\boldsymbol{\Omega}_{k e}^{k}=\left(\begin{array}{ccc}
\cos \gamma & 0 & -\sin \gamma \\
0 & 1 & 0 \\
\sin \gamma & 0 & \cos \gamma
\end{array}\right)\left[\begin{array}{c}
0 \\
0 \\
\dot{\chi}_{k}
\end{array}\right]+\left[\begin{array}{c}
0 \\
\dot{\gamma}_{k} \\
0
\end{array}\right]=\left(\begin{array}{c}
-\sin \gamma \cdot \dot{\chi} \\
\dot{\gamma} \\
\cos \gamma \cdot \dot{\chi}
\end{array}\right)
$$

Combining all previous information leads to the expression:

$$
\begin{aligned}
\frac{d \mathbf{V}_{k}}{d t} & =\left(\begin{array}{c}
\dot{V}_{k} \\
0 \\
0
\end{array}\right)+\left(\begin{array}{c}
-\sin \gamma \cdot \dot{\chi} \\
\dot{\gamma} \\
\cos \gamma \cdot \dot{\chi}
\end{array}\right) \times\left(\begin{array}{c}
V_{k} \\
0 \\
0
\end{array}\right)=\left(\begin{array}{c}
\dot{V}_{k} \\
V_{k} \cdot \cos \gamma \cdot \dot{\chi} \\
-V_{k} \cdot \dot{\gamma}
\end{array}\right) \\
& =\frac{1}{m}\left(\begin{array}{ccc}
1 & 0 & 0 \\
0 & \cos \mu & -\sin \mu \\
0 & \sin \mu & \cos \mu
\end{array}\right)\left(\begin{array}{c}
F_{A_{X}} \\
F_{A_{Y}} \\
F_{A_{Z}}
\end{array}\right)+\left(\begin{array}{c}
-g \sin \gamma \\
0 \\
g \cos \gamma
\end{array}\right)
\end{aligned}
$$

Rewriting this result for $\dot{V}_{k}, \dot{\gamma}$ and $\dot{\chi}$ :

$$
\begin{aligned}
\dot{V}_{k} & =\frac{1}{m} F_{A_{X}}-g \sin \gamma \\
\dot{\chi} & =\frac{1}{m V_{k} \cdot \cos \gamma}\left(\cos \mu \cdot F_{A_{Y}}-\sin \mu \cdot F_{A_{Z}}\right) \\
\dot{\gamma} & =\frac{1}{-m V_{k}}\left(\sin \mu \cdot F_{A_{Y}}+\cos \mu \cdot F_{A_{Z}}\right)-\frac{g \cos \gamma}{V_{k}}
\end{aligned}
$$

Finally, these expressions need to be rewritten for the roll angle $\mu$ and the symmetric aerodynamic forces $F_{A_{X}}$ and $F_{A_{Z}}$. This process is by no means unique, and several ways have been explored to do so. Comparing two mathematical inversions and one goniometrical inversion has pointed out that the latter is preferable, since it is physically intuitive and it does not require solving a quadratic polynomial, in contrast to the two former procedures.

Figure 1 illustrates the forces acting on the reference frame axes perpendicular on the velocity. The magnitude of the total required force, $\left|F_{\text {required }}\right|$ and the required roll angle $\mu_{\text {required }}$ can be derived from this figure. Given that

$$
\begin{aligned}
\left|F_{\text {required }}\right|= & \sqrt{F_{\text {horizontal }}^{2}+F_{\text {vertical }}^{2}} \\
\tan \mu_{\text {required }}= & \frac{F_{\text {horizontal }}}{F_{\text {vertical }}} \\
& 7 \text { of } 17
\end{aligned}
$$




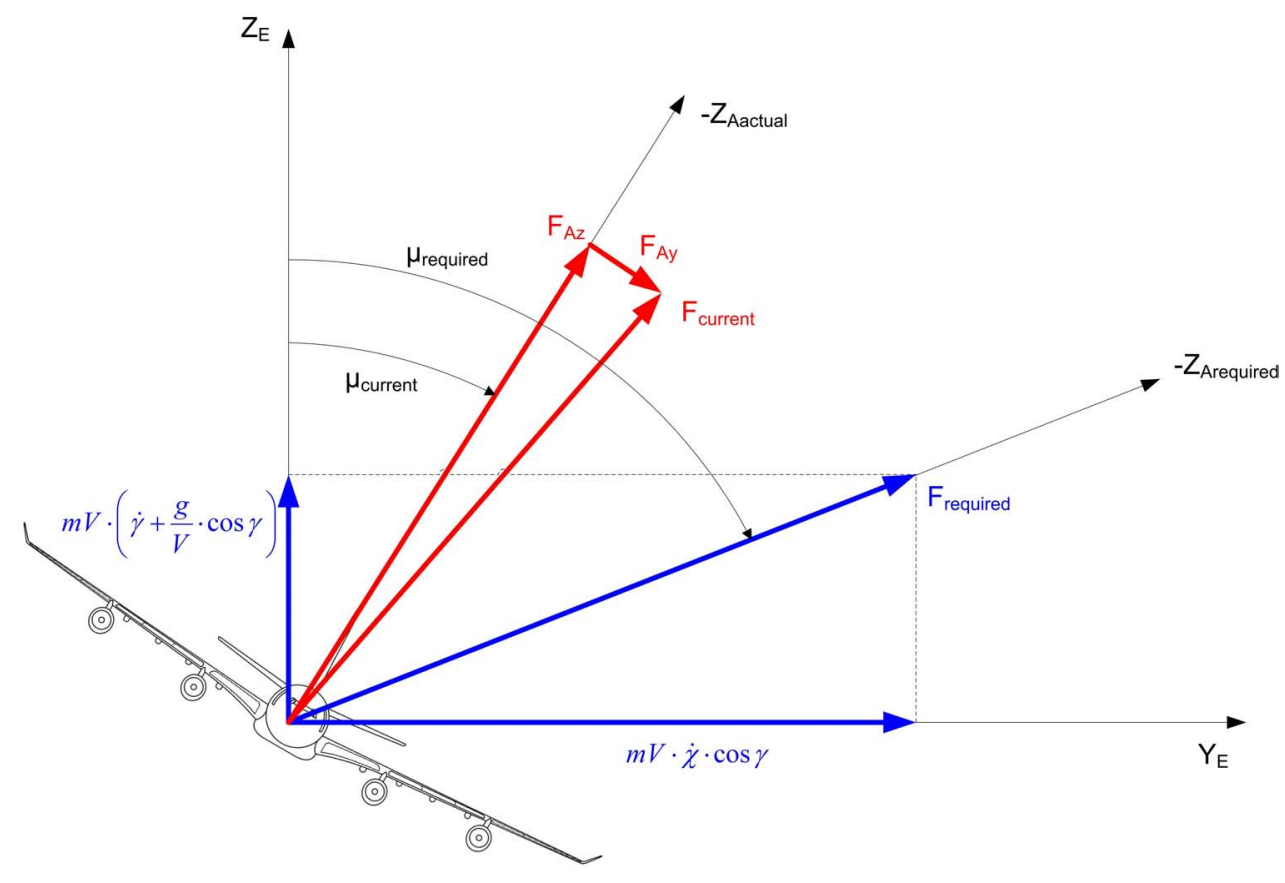

Figure 1. Forces acting on the refererence frame axes in the plane perpendicular on the velocity vector

where these force components are expressed with respect to the earth fixed reference frame. From eq. (37) it is clear that these can be written as follows:

$$
\begin{aligned}
F_{\text {horizontal }} & =m V_{k} \dot{\chi} \cos \gamma \\
F_{\text {vertical }} & =m V_{k} \dot{\gamma}+m g \cos \gamma=m V_{k}\left(\dot{\gamma}+\frac{g}{V_{k}} \cos \gamma\right)
\end{aligned}
$$

Combining this information, while taking into account the contribution from the lateral sideforce $F_{A_{y}}$ results in the required set of quantities:

$$
\begin{aligned}
F_{A_{X}} & =m(\dot{V}+g \sin \gamma) \\
F_{A_{Z}} & =-\cos \gamma \sqrt{m^{2}\left[\left(g+\frac{V \dot{\gamma}}{\cos \gamma}\right)^{2}+(V \dot{\chi})^{2}\right]-\left(\frac{F_{A_{Y}}}{\cos \gamma}\right)^{2}} \\
\mu & =\arctan \left(\frac{\dot{\chi} \cos \gamma}{\dot{\gamma}+g \frac{\cos \gamma}{V}}\right)
\end{aligned}
$$

where $F_{A_{Z}}$ corresponds to $\left|F_{\text {required }}\right|$ and $\mu$ is in fact $\mu_{\text {required }}$.

2. Transformation of force components into commanded angle of attack and dimensionless thrust values

Since the aerodynamic forces are available in the body fixed reference frame, they must be converted towards the aerodynamic reference frame, involving a rotation around the angle of attack $\alpha$ and the sideslip angle $\beta$ :

$$
\begin{aligned}
F_{A} & =\left(\begin{array}{ccc}
\cos \alpha & 0 & \sin \alpha \\
0 & 1 & 0 \\
-\sin \alpha & 0 & \cos \alpha
\end{array}\right)\left(\begin{array}{ccc}
\cos \beta & \sin \beta & 0 \\
-\sin \beta & \cos \beta & 0 \\
0 & 0 & 1
\end{array}\right) F_{B} \\
\left(\begin{array}{c}
F_{A_{X}} \\
F_{A_{Y}} \\
F_{A_{Z}}
\end{array}\right) & =\bar{q} S\left(\begin{array}{c}
\cos \alpha \cos \beta \cdot C_{X}+\cos \alpha \sin \beta \cdot C_{Y}+\sin \alpha \cdot C_{Z} \\
-\sin \beta \cdot C_{X}+\cos \beta \cdot C_{Y} \\
-\sin \alpha \cos \beta \cdot C_{X}-\sin \alpha \sin \beta \cdot C_{Y}+\cos \alpha \cdot C_{Z}
\end{array}\right)
\end{aligned}
$$


The dimensionless force coefficients in the three axes are expanded as follows:

$$
\begin{aligned}
C_{X} & =C_{X_{0}}+C_{X_{\alpha}} \alpha+C_{X_{\alpha^{2}}} \alpha^{2}+C_{X_{q}} \frac{q \bar{c}}{V}+C_{X_{\delta_{e}}} \delta_{e}+C_{X_{T_{c}}} T_{c} \\
& \approx C_{X_{0}}+C_{X_{\alpha}} \alpha+C_{X_{\alpha^{2}}} \alpha^{2}+C_{X_{T_{c}}} T_{c} \\
C_{Y} & =C_{Y_{0}}+C_{Y_{\beta}} \beta+C_{Y_{p}} \frac{p b}{2 V}+C_{Y_{r}} \frac{r b}{2 V}+C_{Y_{\delta_{\alpha}}} \delta_{a}+C_{Y_{\delta_{r}}} \delta_{r} \\
C_{Z} & =C_{Z_{0}}+C_{Z_{\alpha}} \alpha+C_{Z_{q}} \frac{q \bar{c}}{V}+C_{Z_{\delta_{e}}} \delta_{e}+C_{Z_{T_{c}}} T_{c} \approx C_{Z_{0}}+C_{Z_{\alpha}} \alpha
\end{aligned}
$$

It should be noted that the above expansions have been simplified in order to reduce complexity. First of all, the first order Taylor series expansion has been limited to the most important independent variables. For the $X$-force, these are the constant term, angle of attack $\alpha$ and its square $\alpha^{2}$ and the dimensionless thrust coefficient $T_{c}$. For the $Z$-force, only the constant term and angle of attack $\alpha$ are relevant. Although being a considerable simplification, this is justifiable. First of all, angular rates and control surface deflections have a primary influence on the aerodynamic moments. The aerodynamic forces, which always have some delay compared to the moments, depend primarily on the aerodynamic angles and thrust. Moreover, taking out these dependencies eliminates an implicit feedback loop of angular rates and deflections, which would increase the complexity unnecessarily. In case of serious aerodynamic failures, additional regressors can become relevant in these force expressions, but these possible influences can be taken into account selectively by means of Adaptive Recursive Orthogonal Least Squares AROLS. ${ }^{37}$ Finally, the contribution of the sideslip angle $\beta$ in $F_{A_{X}}, F_{A_{Y}}$ and $F_{A_{Z}}$ is not discounted via the dimensionless coefficient $C_{Y}$, but through the specific force $A_{Y}$. Reason for this is that this expression does not need to be rewritten towards $\beta$. Consequently, the dimensionless $Y$-force coefficient can be calculated by means of the specific force in the relevant direction:

$$
Y_{\text {aero }}=m A_{Y}=\bar{q} S C_{Y} \quad \Rightarrow \quad C_{Y}=\frac{m A_{Y}}{\bar{q} S}
$$

Combining this information in eq. (46) results in the following expressions:

$$
\begin{aligned}
& F_{A_{X}}=\bar{q} S\left(\cos \alpha \cos \beta \cdot C_{X}\left(\alpha, T_{C}\right)+\cos \alpha \sin \beta \cdot \frac{m A_{Y}}{\bar{q} S}+\sin \alpha \cdot C_{Z}(\alpha)\right) \\
& F_{A_{Y}}=\bar{q} S\left(-\sin \beta \cdot C_{X}\left(\alpha, T_{C}\right)+\cos \beta \cdot \frac{m A_{Y}}{\bar{q} S}\right) \\
& F_{A_{Z}}=\bar{q} S\left(-\sin \alpha \cos \beta \cdot C_{X}\left(\alpha, T_{C}\right)-\sin \alpha \sin \beta \cdot \frac{m A_{Y}}{\bar{q} S}+\cos \alpha \cdot C_{Z}(\alpha)\right)
\end{aligned}
$$

This system of equations must be rewritten towards the quantities angle of attack $\alpha$ and the dimensionless thrust coefficient $T_{c}$. However, this cannot be done as easily as in the previous section, since higher order influences are present in this system of equations, which is moreover overdetermined. Although it could be argued to ignore the influences of the lateral force in the $Y$-direction on these symmetric quantities for unfailed aircraft, this simplification does not hold here, due to the fact that this control law needs to be applicable for asymmetrically damaged aircraft, possibly flying with nonzero sideslip angle $\beta \neq 0$. Despite the presence of these higher order influences, they are still gradual and relatively small. Therefore, they can be treated globally as linear influences on these forces in the aerodynamic frame of reference:

$$
\begin{aligned}
& F_{A_{X}} \approx F_{A_{X_{0}}}+F_{A_{X_{\alpha}}} \alpha+F_{A_{X_{T c}}} T c \\
& F_{A_{Y}} \approx F_{A_{Y}}+F_{A_{Y_{\alpha}}} \alpha+F_{A_{Y_{T c}}} T c \\
& F_{A_{Z}} \approx F_{A_{Z_{0}}}+F_{A_{Z_{\alpha}}} \alpha+F_{A_{Z_{T c}}} T c
\end{aligned}
$$

These force coefficients are calculated on-line for a realistic range of values for angle of attack and dimensionless thrust, while avoiding collinearities between both ranges. This on-line calculation procedure is called "Local Gradient Determination" (LGD). Extensive validations have shown that these approximations are sufficiently accurate. Finally, the aforementioned structure allows to rewrite the system for angle of attack and dimensionless thrust in the usual structure as used for nonlinear dynamic inversion.

$$
\left(\begin{array}{c}
\alpha \\
T_{C}
\end{array}\right)=\left(\begin{array}{cc}
F_{A_{X_{\alpha}}} & F_{A_{X_{T c}}} \\
F_{A_{Y_{\alpha}}} & F_{A_{Y_{T c}}} \\
F_{A_{Z_{\alpha}}} & F_{A_{Z_{T c}}}
\end{array}\right)^{\dagger}\left[\left(\begin{array}{c}
F_{A_{X_{\mathrm{Xcomm}}}} \\
F_{A_{Y}} \\
F_{A_{Z \mathrm{zcomm}}}
\end{array}\right)-\left(\begin{array}{c}
F_{A_{X_{0}}} \\
F_{A_{Y_{0}}} \\
F_{A_{Z_{0}}}
\end{array}\right)\right]
$$


where the symbol $\dagger$ denotes the left inverse.

Summarizing, the global setup of the third dynamic inversion loop can be found in figure 2. The integration of the second and third inversion loop is illustrated in figure 3.

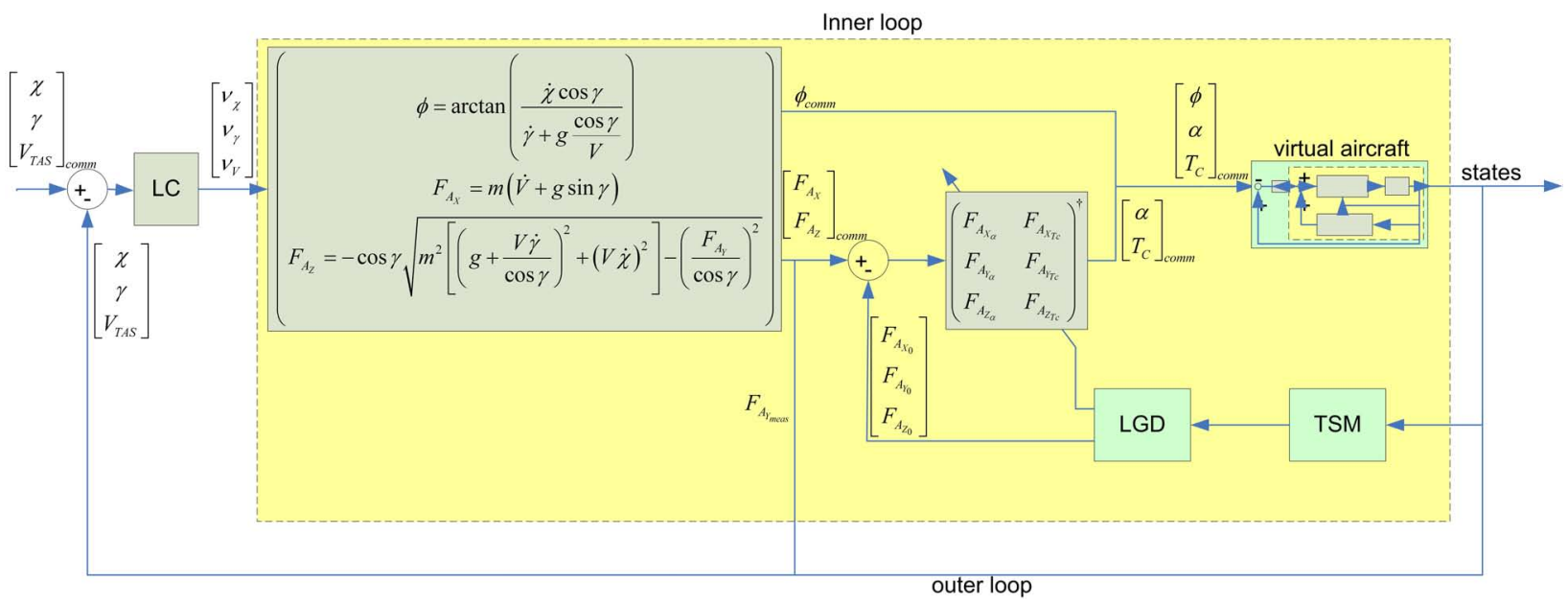

Figure 2. Third NDI autopilot loop, featuring $V_{T A S}, \gamma$ and $\chi$ control. LGD stands for local gradient determination. TSM represents the two step method model identification block elaborated in. ${ }^{8}$

\section{Flying with nonzero sideslip angle to counteract asymmetric damage}

As can be seen in fig. 3, the commanded sideslip angle $\beta_{\text {comm }}$ is zero in all circumstances. However, for asymmetric damage, it might be necessary to fly with a non-zero sideslip angle in order to reach an equilibrium condition. This control setup and the concept of time scale separation take this issue automatically into account. First of all, the yaw rate behavior $r$ of an aircraft is much faster than its slipping behavior in $\beta$. Therefore, after the asymmetric damage abruptly occurs, like in the engine separation scenario, the aircraft will start yawing towards the lost engines and the inner loop will counteract for this response at once. At second instance, while the yawing rotational rate is being reduced to zero, nonzero sideslip is building up gradually. In a classical linear control setup for the middle loop without NDI, this behavior would prevent that a new equilibrium condition could be achieved, because of the continuous nonzero commanded yaw rate $r_{\text {comm }}=-K_{\beta} \beta_{\text {meas }}$. However, thanks to the presence of NDI control in the middle loop, this problem does not occur here, as the commanded yaw rate is calculated as follows:

$$
r_{\mathrm{comm}} \approx\left(-\frac{u}{\sqrt{V^{2}-v^{2}}}\right)^{-1} \cdot\left[-K_{\beta} \beta_{\text {meas }}-\frac{1}{\sqrt{V^{2}-v^{2}}}\left(A_{y}+g \cos \theta \sin \phi\right)\right]
$$

It should be noted that this equation is simplified for transparency by ignoring the contribution from the virtual roll angle input $\nu_{\dot{\phi}}$. This contribution is in fact small compared to the other terms, but in the control setup this input does contribute effectively, as can be seen in fig. 3. Eq. (58) shows that $r_{\text {comm }}$ returns to zero when the nonzero values of sideslip $\beta_{\text {meas }}$, lateral specific force $A_{y}$ and roll angle $\phi$ come in balance, after the faster dynamics of the yaw rate have damped out.

\section{Pseudo Control Hedging Principle}

The concept of Pseudo Control Hedging (PCH) was initially developed by Johnson. ${ }^{24,25}$ Aim is to compensate the reference model signal for input characteristics such as actuator position limits, actuator rate limits, and linear input dynamics. In the work of Holzapfel, ${ }^{40}$ this concept has been applied in an adaptive control setup based upon nonlinear dynamic inversion. An additional advantage of $\mathrm{PCH}$ is that the relative order of the dynamic inversion operation can be reduced by one degree. NDI inverts the aircraft dynamics, but not the input behavior, represented by the actuators, which are usually modelled as first order lag components. The use of $\mathrm{PCH}$ provides an alternative for an eventual additional inversion loop for these dynamics, although this has been no point of concern in the research setup presented here. Nevertheless, 


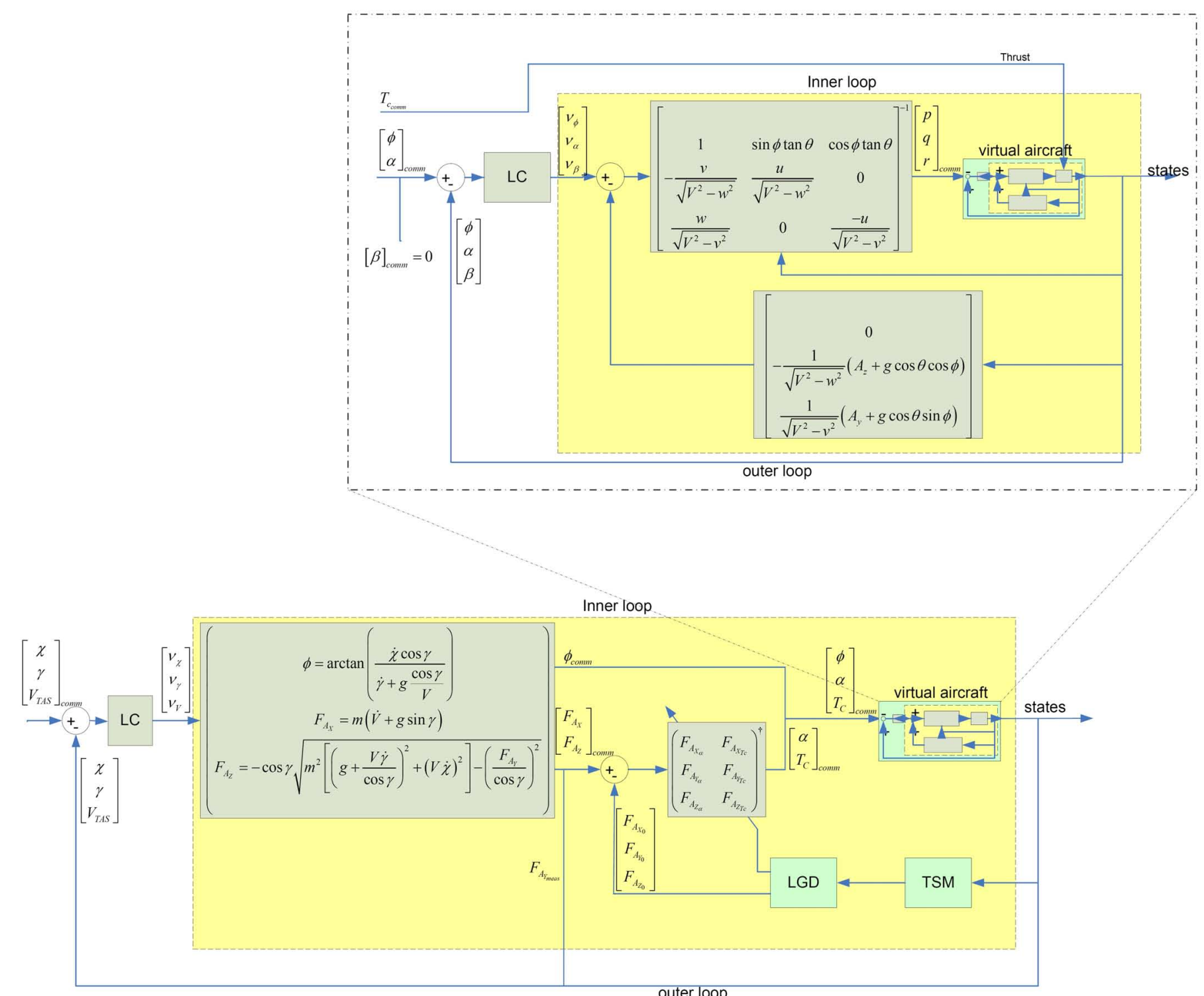

Figure 3. Assembled NDI autopilot setup, with second and third NDI loop portrayed in detail. 
input saturation is a concern for nonlinear dynamic inversion based control.

The principle of Pseudo Control Hedging works as follows. For system dynamics of the form: $\dot{\mathbf{x}}=\mathbf{a}(\mathbf{x})+$ $\mathbf{b}(\mathbf{x}) \boldsymbol{\delta}_{\text {comm }}$, where $\mathbf{a}(\mathbf{x})$ contains aerodynamic model information and $\mathbf{b}(\mathbf{x})$ represents control effectivity, the NDI control law is structured as follows: $\boldsymbol{\delta}_{\text {comm }}=\mathbf{b}^{-1}(\mathbf{x})[\boldsymbol{\nu}-\mathbf{a}(\mathbf{x})]$, resulting in: $\dot{\mathbf{x}}=\boldsymbol{\nu}$, where $\boldsymbol{\nu}$ is defined as the virtual input. Due to the control effector characteristics, such as actuator position and rate limits, the actual control displacement $\boldsymbol{\delta}_{\text {act }}$ is not identical to the commanded control displacement $\boldsymbol{\delta}_{\text {comm. }}$. Subsequently, the estimated virtual input $\hat{\boldsymbol{\nu}}$ can be calculated, which is based upon the actual control displacement $\boldsymbol{\delta}_{\text {act }}$ :

$$
\hat{\boldsymbol{\nu}}=\mathbf{a}(\mathbf{x})+\mathbf{b}(\mathbf{x}) \boldsymbol{\delta}_{\text {act }}
$$

Finally, the PCH signal $\boldsymbol{\nu}_{h}$ is obtained by subtracting the estimated from the commanded virtual input:

$$
\boldsymbol{\nu}_{h}=\boldsymbol{\nu}-\hat{\boldsymbol{\nu}}
$$

This signal serves as compensation signal which is fed back to the reference model and which is subtracted from $\dot{\mathbf{x}}_{\text {ref. }}$ The reference signal at the output of the reference model becomes:

$$
\mathbf{x}_{\mathrm{comm}}=\frac{1}{s}\left(\mathbf{K}_{\mathrm{ref}}\left(\mathbf{x}_{\mathrm{ref}}-\mathbf{x}_{\mathrm{comm}}\right)-\boldsymbol{\nu}_{h}\right)
$$

The overview of the Pseudo Control Hedging setup can be seen in fig. 4. This figure shows clearly that $\mathrm{PCH}$ scales (hedges) the commanded signal down to a level that is achievable by the actuator dynamics.

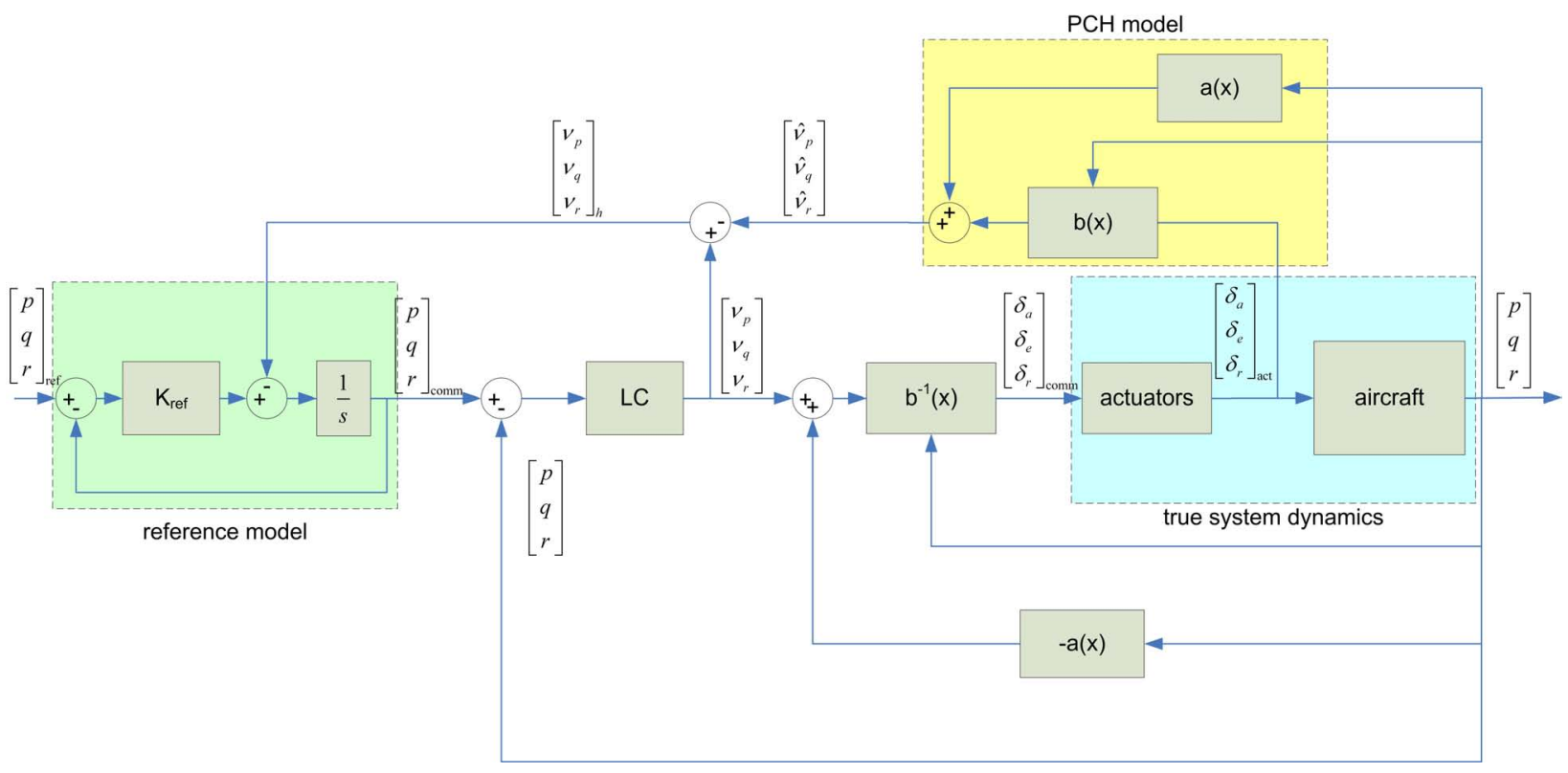

Figure 4. Setup of the Pseudo Control Hedging structure

The advantage of this setup is that for adaptive NDI, damage information in the identified aerodynamic model as well as in the control surface deflections is automatically taken into account while calculating the estimated virtual input $\hat{\nu}$ and subsequently the PCH signal $\nu_{h}$. The beneficial influence of PCH for this purpose is illustrated in the evaluation results presented next.

\section{Applications}

The combined setup of NDI and PCH has been evaluated on a high fidelity Pratt and Whitney powered Boeing 747-100/200 simulation model. A detailed nonlinear simulation model of this aircraft is available from the National Aerospace Laboratory NLR. This RECOVER (REconfigurable COntrol for Vehicle Emergency Relief) benchmark model is discussed in detail by Smaili et $\mathrm{al}^{26,27}$ 
As explained in section II, the improved control setup consists of three consecutive NDI loops. This means that $\mathrm{PCH}$ can be implemented on these three levels as well. Application is rather straightforward by combining the PCH laws eq. (59), (60) and (61) from the previous section and the NDI control laws as given in eq. (15), (26) and (44) combined with eq. (57) for the inner, middle and outer loops respectively. Two application examples are given below, a pitch rate example in the inner loop and a velocity example in the outer loop.

\section{A. pitch rate channel}

In the first example, a doublet command is given on the pitch rate channel, while two of the four elevators are defective, i.e. they are jammed in trim position. The saturation limits of the non-defective elevators are $+17 /-23$ deg. Figure 5 illustrates the consequences of actuator saturation without pseudo-control hedging. Fig. 5(b) shows that elevator deflection angle limit saturation occurs between $60 \mathrm{~s}$ and $65 \mathrm{~s}$. As a consequence, the reference pitch rate cannot be achieved by the measured pitch rate, as shown in fig. 5(a). The beneficial influence of $\mathrm{PCH}$ is visualized in figure 6 . The protecting effect of the $\mathrm{PCH}$ signal in the reference model can be seen in fig. 6(a), where the hedged reference signal $q_{\mathrm{ref}_{\mathrm{PCH}}}$ differs significantly from the regular doublet reference signal $q_{\text {ref }}$. Figure $6(\mathrm{~b})$ shows that the control surface does not saturate anymore, and as a consequence the measured pitch rate $q_{\text {meas }}$ matches the compensated reference signal $q_{\text {ref }}$ PCH in fig. 6(a). Comparing fig. 5 and 6 illustrates that actuator rate saturation is responsible for the initial hedging signal, later on augmented by the position saturation.

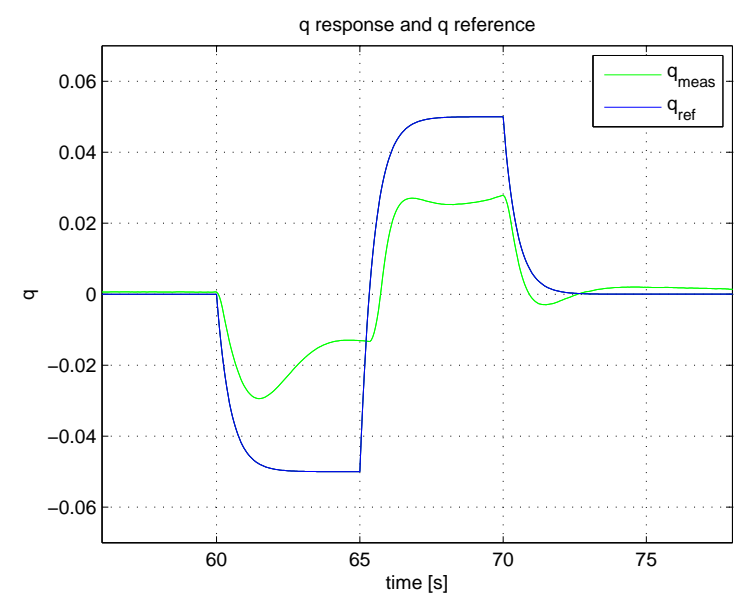

(a) pitch rate response and pitch rate reference

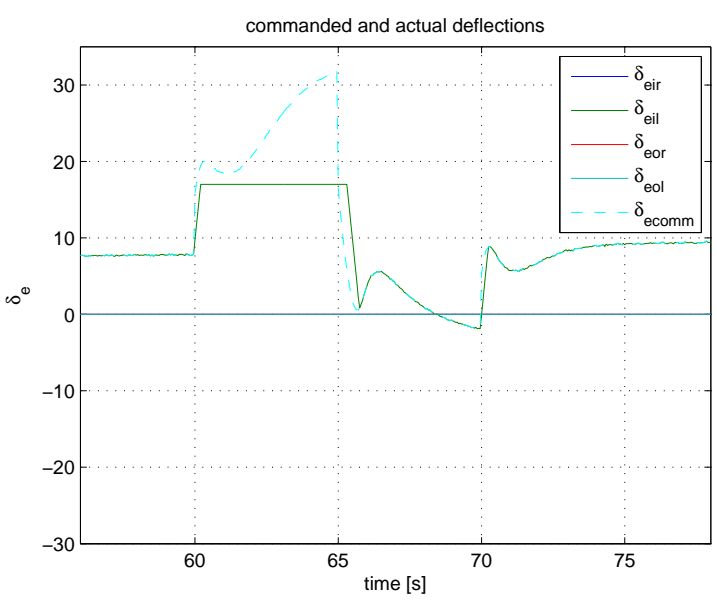

(b) commanded and actual deflections

Figure 5. Pitch rate doublet command without $\mathrm{PCH}$

This basic example illustrates the use of $\mathrm{PCH}$ in the control loop. However, it is possible to implement this algorithm on all control layers, and this overall application is illustrated by the following example.

\section{B. velocity channel}

In this example, a step command is given on the velocity channel combined with an altitude change, while some performance degrading restrictions apply on the engine parameters, i.e. they react slower and can give less maximum thrust. As a consequence, for the situation without $\mathrm{PCH}$ in figure $7(\mathrm{a})$, it can be seen that the slower engine reactions lead to a longer rise time and considerable overshoot. Comparing thrust command and actual thrust values in fig. 7(b) shows that saturation occurs over sustained periods of time. In contrast, figure 8 shows the effect of $\mathrm{PCH}$. In figure $8(\mathrm{a}), \mathrm{PCH}$ provides a corrected reference signal $V_{\text {ref }_{\mathrm{PCH}}}$ which is reachable in the present configuration. The hedging effect on the reference signal is caused by the simultaneous altitude change, which requires an amount of the restricted available remaining thrust authority to achieve. As soon as the altitude change has been achieved, between 400 and 500s, the hedging influence disappears. However, figure $8(\mathrm{~b})$ shows that thrust saturation still occurs, although the level of saturation and its time span are considerably reduced, as can be seen by comparing with fig. 7(b). Further 


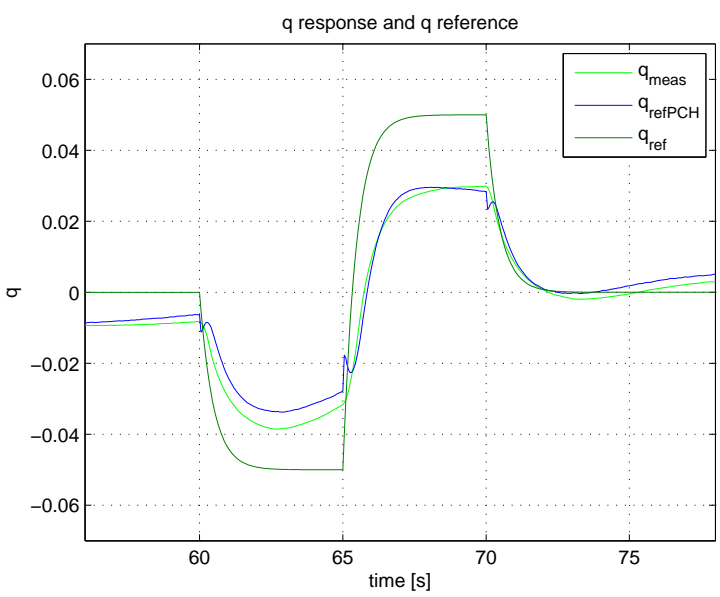

(a) pitch rate response and pitch rate reference

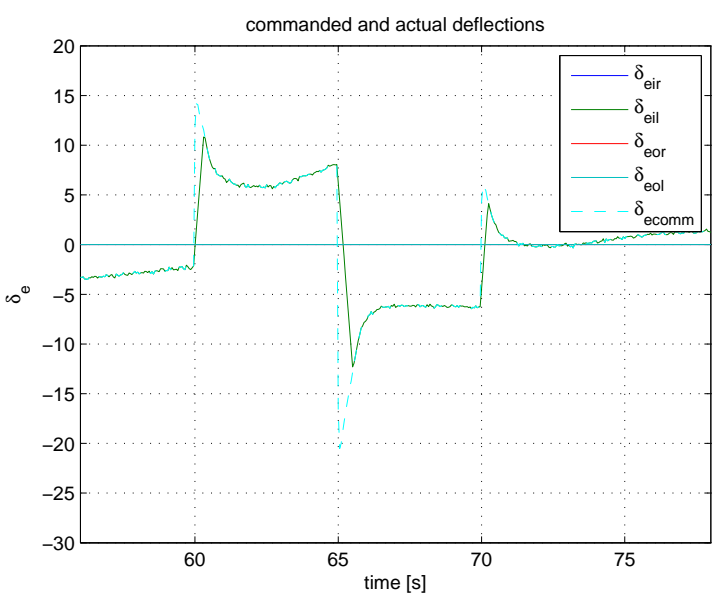

(b) commanded and actual deflections

Figure 6. Pitch rate doublet command with $\mathrm{PCH}$

analysis has shown that saturation occurs in the time intervals when relatively small fit errors occur in the local gradient calculation of the third NDI loop, as described in subsection 2 of section II. This result shows that very accurate identification results are needed for accurate PCH which eliminates all saturation in the controls. However, even with small misfits in the local gradient determination results, the beneficial influence of $\mathrm{PCH}$ is still clear as can be seen by comparing figures $7(\mathrm{~b})$ and $8(\mathrm{~b})$.

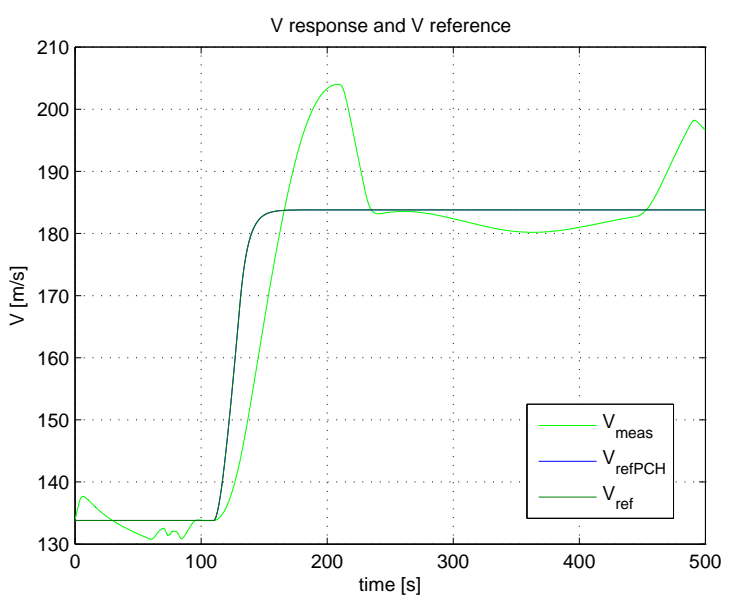

(a) velocity response and velocity reference

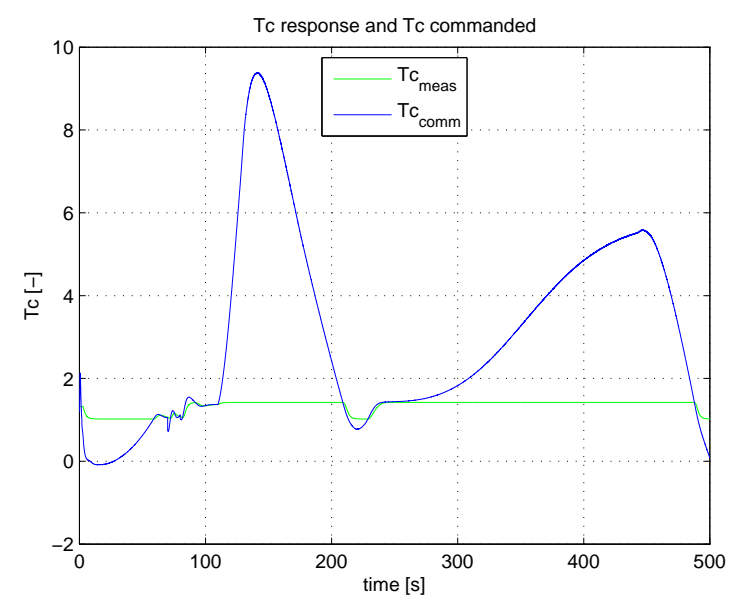

(b) commanded and actual thrust

Figure 7. Velocity change command with engine limitations without PCH

\section{Conclusions and remarks}

A major conclusion from these simulations is that avoiding control saturation, especially with respect to the throttles, is a crucial aspect to guarantee survivability in post failure conditions. For this purpose, Pseudo Control Hedging has been implemented. A major advantage of the combination of NDI and PCH is their physical meaning, which greatly enhances the interpretability of all signals.

Analysis of the simulation results as presented above has shown that avoiding control saturation on all levels of the dynamic inversion controller by means of pseudo control hedging is an important part of 


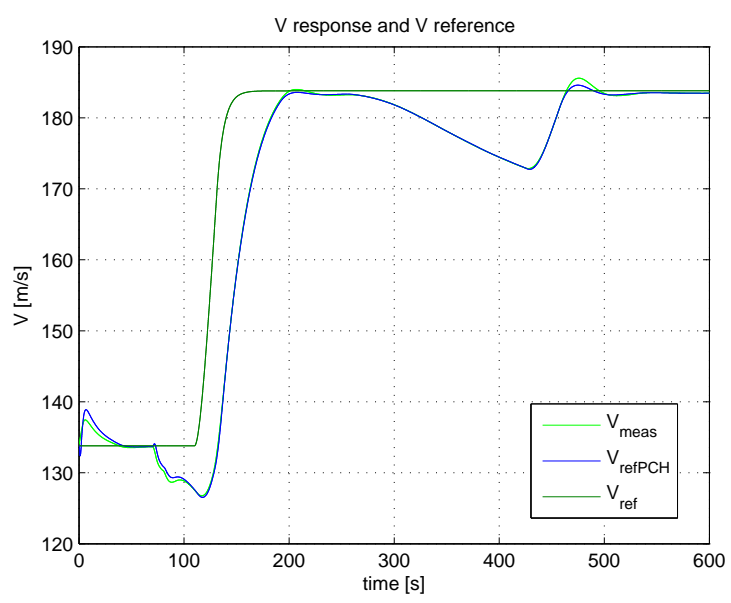

(a) velocity response and velocity reference

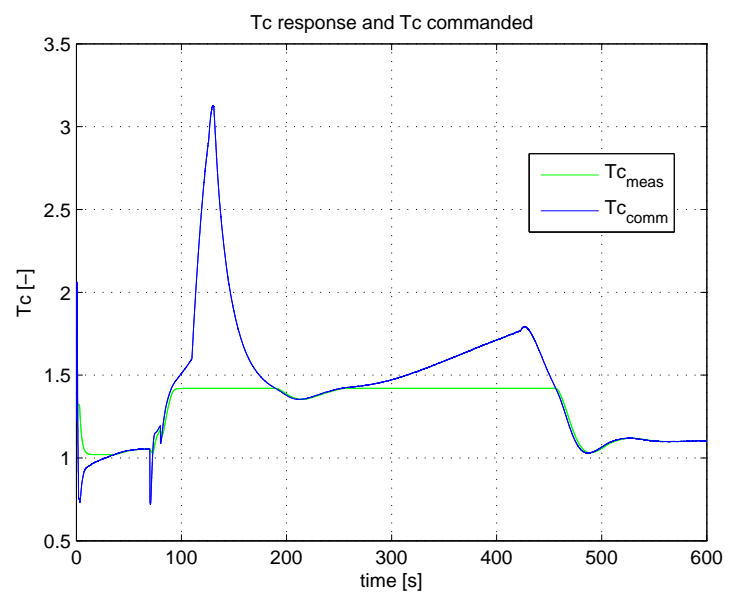

(b) commanded and actual thrust

Figure 8. Velocity change command with engine limitations with PCH

safe flight envelope protection. However, this cannot be considered as a complete protection algorithm. This routine focuses exclusively on the avoidance of input saturation, which plays an important role in this topic. There are also other aspects, like minimum control airspeed, maximum roll angle etc., which are not considered by PCH. Moreover, the current set-up of $\mathrm{PCH}$ does not yet prioritize e.g. speed $V$ over flight path angle $\gamma$, as can be seen in fig. 8. As a result, it is recommended to impose limitations inside the reference model for safe extreme values of the flight path angle based upon the true airspeed.

\section{Appendix: Frame conversions}

Figure 9 illustrates the matrix transformations which are needed to make the conversions from one reference frame to the other.

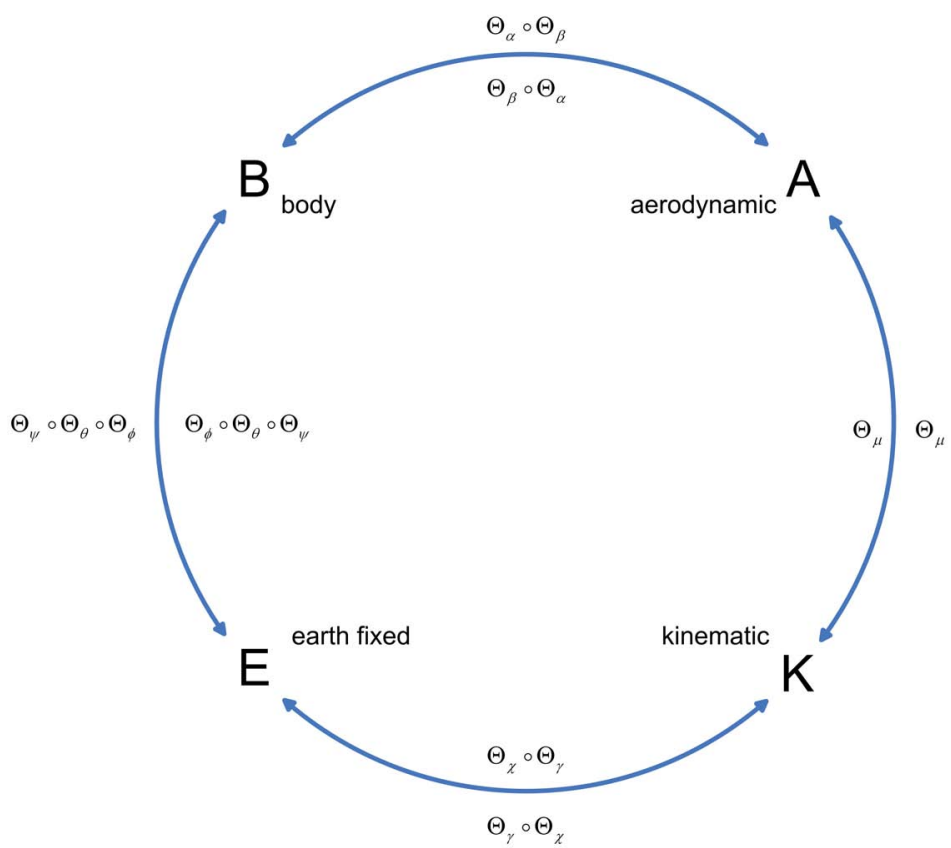

Figure 9. Reference frame conversions. Inner sequences apply for clockwise directions, outer for counterclockwise directions 


\section{Acknowledgments}

The availability of a trustworthy simulation model, was a very important contribution to this study. This model, which was developed by Hafid Smaili and Jan Breeman from the Dutch Aerospace Laboratory (NLR), provided an essential instrument in order to test the ability of the FTFC to deal with a damaged aircraft model in some realistic failure scenarios. This research was supported by the Dutch Technology Foundation (STW) under project number 06515 and has been performed during a research visit at the department of Robotics and Mechatronics at the German aerospace center DLR in Oberpfaffenhofen.

\section{References}

${ }^{1}$ Dutoi, B., Richards, N., Gandhi, N., Ward, D., and Leonard, J., "Hybrid Robust Control and Reinforcement learning for Optimal Upset Recovery," AIAA Guidance, Navigation and Control Conference and Exhibit, Honolulu, HI, August 18-21 2008.

${ }^{2}$ Litt, J. and Guo, T., "Fast Thrust Response for Improved Flight/Engine Control under Emergency Conditions," AIAA Guidance, Navigation and Control Conference and Exhibit, Honolulu, HI, August 18-21 2008.

${ }^{3}$ Boskovic, J., Knoebel, N., Mehra, R., and Gregory, I., "An Integrated Approach to Damage Accommodation in Flight Control," AIAA Guidance, Navigation and Control Conference and Exhibit, Honolulu, HI, August 18-21 2008.

${ }^{4}$ Tang, L., Roemer, M., Bharadwaj, S., and Belcastro, C., "An Integrated Health Assessment and Fault Contingency Management System for Aircraft," AIAA Guidance, Navigation and Control Conference and Exhibit, Honolulu, HI, August $18-212008$

${ }^{5}$ Chang, B., Kwatny, H., Belcastro, C., and Belcastro, C., "Aircraft Loss-of-Control Accident Prevention: Switching Control of the GTM Aircraft with Elevator Jam Failures," AIAA Guidance, Navigation and Control Conference and Exhibit, Honolulu, HI, August 18-21 2008.

${ }^{6}$ Bosworth, J., "Flight Results of the NF-15B Intelligent Flight Control System (IFCS) Aircraft with Adaptation to a Longitudinally Destabilized Plant," AIAA Guidance, Navigation and Control Conference and Exhibit, Honolulu, HI, August 18-21 2008

${ }^{7}$ Johnson, E., Calise, A. J., and De Blauwe, H., "In Flight Validation of Adaptive Flight Control Methods," AIAA Guidance, Navigation and Control Conference and Exhibit, Honolulu, HI, August 18-21 2008.

${ }^{8}$ Lombaerts, T., Huisman, H., Chu, Q., Mulder, J., and Joosten, D., "Nonlinear Reconfiguring Flight Control based on On-line Physical Model Identification," AIAA Journal of Guidance, Control and Dynamics, Vol. 32, No. 3, May-June 2009 2009, pp. $727-748$.

${ }^{9}$ Sonneveldt, L., van Oort, E., Chu, Q., and Mulder, J., "Nonlinear Adaptive Trajectory Control Applied to an F-16 Model," AIAA Journal of Guidance, Control and Dynamics, Vol. 32, No. 1, January-February 2009, pp. 25-39.

${ }^{10}$ Maciejowski, J. and Jones, C., "MPC Fault Tolerant Flight Control Case Study: Flight 1862," IFAC Safeprocess Conference, 2003.

${ }^{11}$ Joosten, D., van den Boom, T., and Lombaerts, T., "Computationally Efficient use of MPC and Dynamic Inversion for Reconfigurable Flight Control," AIAA Guidance, Navigation and Control Conference and Exhibit, No. AIAA-2008-7431, Honolulu, HI, August 18-21 2008.

${ }^{12}$ Walker, G. and Allen, D., "X-35B STOVL Flight Control Law Design and Flying Qualities," Proceedings of the Biennial International Powered Lift Conference and Exhibit, No. AIAA-2002-6018, 2002.

${ }^{13}$ Balas, G., "Flight Control Law Design: An Industry Perspective," European Journal of Control, special issue, Vol. 9, No. 2-3, 2003, pp. 207-226.

${ }^{14}$ Campa, G., Seanor, B., Gu, Y., and Napolitano, M. R., "NLDI Guidance Control Laws for Close Formation Flight," American Control Conference, Portland, OR, USA, June 8-10 2005.

${ }^{15}$ Reiner, J., Balas, G. J., and Garrard, W. L., "Flight Control Design Using Robust Dynamic Inversion and Time-scale Separation," Automatica, Vol. 32, No. 11, 1996, pp. 1493-1504.

${ }^{16}$ Ramakrishna, V., Hunt, L., and Meyer, G., "Parameter variations, relative degree, and stable inversion," Automatica, Vol. 37, 2001, pp. 871-880.

${ }^{17}$ Balas, G., Garrard, W., and Reiner, J., "Robust Dynamic Inversion Control Laws for Aircraft Control," Proceedings of the AIAA Guidance, Navigation and Control Conference, AIAA, Washington, DC, 1992, pp. 192-205.

${ }^{18} \mathrm{da}$ Costa, R., Chu, Q. P., and Mulder, J. A., "Re-entry flight controller design using nonlinear dynamic inversion," Journal of Spacecraft and Rockets, Vol. 40, No. 1, 2003, pp. 64-71.

19 van Soest, W., Chu, Q. P., and Mulder, J. A., "Combined feedback linearization and model predictive control for re-entry flight," AIAA Journal of Guidance, Control and Dynamics, Vol. 29, No. 2, 2006, pp. 427-434.

${ }^{20}$ Ostroff, A. J. and Bacon, B. J., "Enhanced NDI strategies for reconfigurable flight control," Proceedings of the American Control Conference, Anchorage, AK, May 8-10 2002.

${ }^{21}$ Ganguli, S., Papageorgiou, G., Glavaski, S., and M., E., "Piloted Simulation of Fault Detection, Isolation and Reconfiguration Algorithms for a Civil Transport Aircraft," AIAA Guidance, Navigation and Control Conference and Exhibit, No. AIAA-2005-5936, San Francisco, CA, August 2005.

${ }^{22}$ Ganguli, S., Papageorgiou, G., Glavaski, S., and M., E., "Aircraft Fault Detection, Isolation and Reconfiguration in the Presence of Measurement Errors," AIAA Guidance, Navigation and Control Conference and Exhibit, No. AIAA-2006-6551, Keystone, Co, August 2006.

${ }^{23}$ Oppenheimer, M. and Doman, D., "Efficient Reconfiguration and Recovery from Damage for Air Vehicles," Proceedings of the AIAA Guidance, Navigation and Control Conference and Exhibit, No. AIAA-2006-6552, 2006. 

2000.

${ }^{24}$ Johnson, E. N., Limited Authority Adaptive Flight Control, Ph.D. thesis, Georgia Institute of Technology, November

${ }^{25}$ Johnson, E. N. and Calise, A. J., "pseudo-control hedging: a new method for adaptive control," Advances in Navigation Guidance and Control Technology Workshop, 2000.

${ }^{26}$ Smaili, M., Breeman, J., Lombaerts, T., and Joosten, D., "A Simulation Benchmark for Integrated Fault Tolerant Flight Control Evaluation," AIAA MST, 2006.

${ }^{27}$ Smaili, M., Breeman, J., and Lombaerts, T., "A Simulation Benchmark for Aircraft Survivability Assessment," 26th International Congress of the Aeronautical Sciences, No. ICAS 2008-9.3.2, Anchorage, AK, 2008.

${ }^{28}$ Marcos, A. and Balas, G., "A Boeing 747-100/200 Aircraft Fault Tolerant and Fault Diagnostic Benchmark," Technical Report AEM-UoM-2003-1, June 2003.

${ }^{29}$ Szaszi, I., Ganguli, S., Marcos, A., Balas, G. J., and Bokor, J., "Application of FDI to a Nonlinear Boeing 747 Aircraft," 10th Mediterranean Conference on Control and Automation, Lisbon, Portugal, July 2002.

${ }^{30}$ Joosten, D., van den Boom, T., and Lombaerts, T., "Effective control allocation in fault-tolerant flight control with MPC and feedback linearization," Proceedings of the European Conference on Systems and control, Kos, Greece, July 2007, pp. 3552-3559.

${ }^{31}$ Joosten, D., van den Boom, T., and Lombaerts, T., "Fault-tolerant control using dynamic inversion and model-predictive control applied to an aerospace benchmark," the Proceedings of the 17th IFAC world congress, 2008, Accepted for presentation at the IFAC world congress.

${ }^{32}$ Cieslak, J., Henry, D., Zolghadri, A., and Goupil, P., "Development of an active fault-tolerant flight control strategy," AIAA Journal of Guidance, Control and Dynamics, Vol. 31, 2008, pp. 135-147.

${ }^{33}$ Hallouzi, R. and Verhaegen, M., "Fault-Tolerant Subspace Predictive Control Applied to a Boeing 747 model," AIAA Journal of Guidance, Control and Dynamics, Vol. 31, 2008, pp. 873-883.

${ }^{34}$ Alwi, H., Edwards, C., Stroosma, O., and Mulder, J., "Fault Tolerant Sliding Mode Control Design with Piloted Simulation Evaluation," AIAA Journal of Guidance, Control and Dynamics, Vol. 31, 2008, pp. 1186-1201.

${ }^{35}$ Edwards, C., Lombaerts, T., and Smaili, M., Fault tolerant control a benchmark challenge, Lecture Notes in Control and Information Sciences, Springer-Verlag, 2010.

${ }^{36}$ Lombaerts, T., Smaili, M., Stroosma, O., Chu, Q., Mulder, J., and Joosten, D., "Piloted Simulator Evaluation Results of New Fault-Tolerant Flight Control Algorithm," AIAA Journal of Guidance, Control and Dynamics, Vol. 32, No. 6, NovemberDecember 2009, pp. 1747-1765.

${ }^{37}$ Lombaerts, T., Van Oort, E., Chu, Q., Mulder, J., and Joosten, D., "Online Aerodynamic Model Structure Selection and Parameter Estimation for Fault-Tolerant Control," Journal of Guidance, Control and Dynamics, 2010, to be published.

${ }^{38} \mathrm{Chu}, \mathrm{Q}$., Lecture Notes AE4-394, Modern Flight Test Technologies and System Identification, Delft University of Technology, Faculty of Aerospace Engineering, 2007.

${ }^{39}$ Slotine, J.-J. E. and Li, W., Applied Nonlinear Control, Prentice Hall, 1991.

${ }^{40}$ Holzapfel, F., Nichtlineare adaptive Regelung eines unbemannten Fluggertes, Ph.D. thesis, Lehrstuhl fr Flugmechanik und Flugregelung, Technische Universitt Mnchen, 2004.

${ }^{41}$ Mulder, J., van Staveren, W., van der Vaart, J., and de Weerdt, E., AE3-302 Flight Dynamics, Lecture Notes, Delft University of Technology, Faculty of Aerospace Engineering, Delft, The Netherlands, January 252006.

${ }^{42}$ Holzapfel, F., "Dynamic Inversion Based Control Concept with Application to an Unmanned Aerial Vehicle," AIAA Guidance, Navigation, and Control Conference and Exhibit, No. AIAA-2004-4907, 2004. 